Research Paper

\title{
Can American Joint Committee on Cancer prognostic groups be individualized in patients undergoing surgery for Stage IV invasive upper tract Urothelial Carcinoma?
}

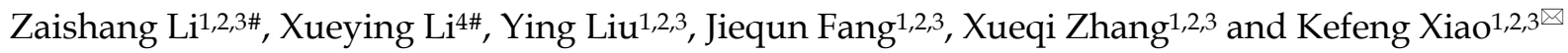 \\ 1. Department of Urology, Shenzhen People's Hospital, The Second Clinic Medical College of Jinan University 518060, Shenzhen, Guangdong, P. R. China. \\ 2. Department of Urology, First Affiliated Hospital of Southern University of Science and Technology, 518060, Shenzhen, Guangdong, P. R. China. \\ 3. Department of Urology, Minimally Invasive Urology of Shenzhen Research and Development Center of Medical Engineering and Technology, 518060, \\ Shenzhen, Guangdong, P. R. China. \\ 4. Department of Oncology, The Seventh Affiliated Hospital Sun Yat-sen University, 518107, Shenzhen, Guangdong, P. R. China. \\ \#These authors contributed equally to this research.
}

$\triangle$ Corresponding author: Ke-Feng Xiao, M.D., Ph.D., TEL.: 86-0755-25533018; FAX: 0755-25533497; E-mail: kevin5510315@qq.com; Address: Department of Urology, Shenzhen People's Hospital, 518060, Shenzhen, Guangdong, P. R. China.

(1) The author(s). This is an open access article distributed under the terms of the Creative Commons Attribution License (https://creativecommons.org/licenses/by/4.0/). See http://ivyspring.com/terms for full terms and conditions.

Received: 2020.07.07; Accepted: 2021.01.06; Published: 2021.02.02

\begin{abstract}
Purpose: We explored whether the modified American Joint Committee on Cancer tumor-nodemetastasis prognostic stage group IV can be individualized in a large population-based cohort of surgically treated invasive upper tract urothelial carcinoma (UTUC) patients.

Methods: Invasive UTUC patients from the Surveillance, Epidemiology and End Results database (2004-2015) were screened for inclusion. A total of 10,482 eligible cases were identified. Cancer-specific survival (CSS) after surgery was analyzed using Kaplan-Meier plots.

Results: According to the most recent pathological prognostic group classification, the 5-year mortality rates of T4NxM0 $(n=493), \operatorname{TxN1M0}(n=597), \operatorname{TxN2M0}(n=424)$ and $\mathrm{pTxNxM1}(n=677)$ patients were $41.1 \%(95 \% \mathrm{Cl} 35.2 \%$ to $47.0 \%), 38.6 \%(95 \% \mathrm{Cl} 33.1 \%$ to $44.1 \%), 40.4 \%(95 \% \mathrm{Cl} 33.0 \%$ to $47.8 \%)$ and $14.2 \%(95 \% \mathrm{Cl} 9.9 \%$ to $18.5 \%$ ), respectively (T4N0M0 vs. TxNxM1, P<0.001; TxN1M0 vs. TxNxM1, $P<0.001$; TxN2M0 vs. TxNxM1, $P<0.001)$. Stage IV tumors were subdivided on the basis of the mortality data (Modification 1): stage IVa tumors were considered nonmetastatic (T4NxM0, TxN1-2M0; 5-year CSS 39.9\%), and stage IVb tumors were considered metastatic (pTxNxMI; 5-year CSS 14.2\%). Stage IV tumors were also subdivided according to the grade classification (Modification 2): stage IVa tumors were

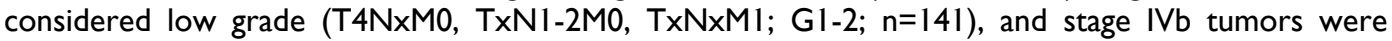
considered metastatic (T4NxM0, TxN1-2M0, TxNxM1; G3-4; $\mathrm{n}=2050$ ). The 5-year CSS rates for stage $\mathrm{IVa}$ and $\mathrm{IVb}$ patients were $76.3 \%(95 \% \mathrm{Cl} 68.7 \%$ to $83.9 \%)$ and $31.4 \%(95 \% \mathrm{Cl} 28.5 \%$ to $34.3 \%)$, respectively $(P<0.001)$.

Conclusions: Stage IV patients were stratified into two prognostically different risk groups depending on metastasis or grade. The subclassification of stage IV can increase the level of prognostic detail and individualize the prediction of survival in invasive UTUC patients.
\end{abstract}

Key words: urothelial carcinoma; upper urinary tract; tumor-node-metastasis; mortality; survival

\section{Introduction}

Urothelial carcinomas are the fourth most common tumors [1]. The American Joint Committee on Cancer/Union Internationale Contre le Cancer (AJCC/UICC) tumor-node-metastasis (TNM) staging system is widely used to predict upper tract urothelial carcinoma (UTUC) patient prognosis, guide treatment options, and evaluate treatment results from different centers. UTUCs are uncommon and account for only $5-10 \%$ of urothelial carcinomas; however, $60 \%$ of UTUCs progress to invasive disease [2]. Because of 
the rarity of this type of cancer, relatively limited data regarding TNM staging have been obtained by the European Association of Urology (EAU) and the National Comprehensive Cancer Network (NCCN) $[2,3]$.

Several studies have verified the validity of the prognostic group classifications [2, 4-6]. In 2017, the $\mathrm{N} 2$ and N3 classifications were redefined in the $8^{\text {th }}$ revision of the TNM staging system [7]. However, the AJCC prognostic group classifications have remained almost unchanged; stage IV still includes $\mathrm{T} 4, \mathrm{~N}+$ and M1 [7]. Therefore room for improvement still exists in the AJCC prognostic group classifications $[8,9]$.

In this study, we critically analyzed the controversial areas of the AJCC-TNM prognostic group staging system to discuss whether the modified AJCC-TNM prognostic stage group IV can be subdivided to provide more detailed prognoses for patients with invasive UTUC.

\section{Materials and methods}

Patients diagnosed with UTUC (ICD-O-2 C65.9 and C66.9 codes) with available TNM stage classification information between 2004 and 2015 were identified from 18 Surveillance, Epidemiology and End Results (SEER) registries. Data from Alaska; Atlanta; California, excluding San Francisco (SF)/San Jose/Monterey (SJM)/Los Angeles (LA); Connecticut; Detroit; Greater Georgia; Hawaii; Kentucky; Los Angeles; Rural Georgia; Louisiana; Iowa; New Jersey; New Mexico; San Francisco; San Jose; Seattle; and Utah were obtained. The characteristics of the SEER population are comparable to those of the general population of the United States.

Among the included patients, only patients with transitional cell carcinoma or papillary transitional cell carcinoma confirmed by histology were considered. The inclusion and exclusion criteria are shown in Fig. 1. The cause of death (cancer-specific versus noncancer related) was defined according to the SEER assignment of the cause of death. Patients who did not die of UTUC were considered to have died due to other causes. The histopathological data were reviewed by an independent pathology committee, and all histopathological reports were based on the AJCC-TNM staging system [7]. For repeated data, the highest level of histopathological data or prime data were considered.

The outcome of interest was cancer-specific survival (CSS). The Kaplan-Meier method was used to determine the CSS. The log-rank test was used to compare CSS rates between different groups. Statistical analyses were performed with Statistical Package for the Social Sciences software (SPSS version 23, IBM Corp, Armonk, NY). A two-sided $P$ value less than 0.05 was considered statistically significant.

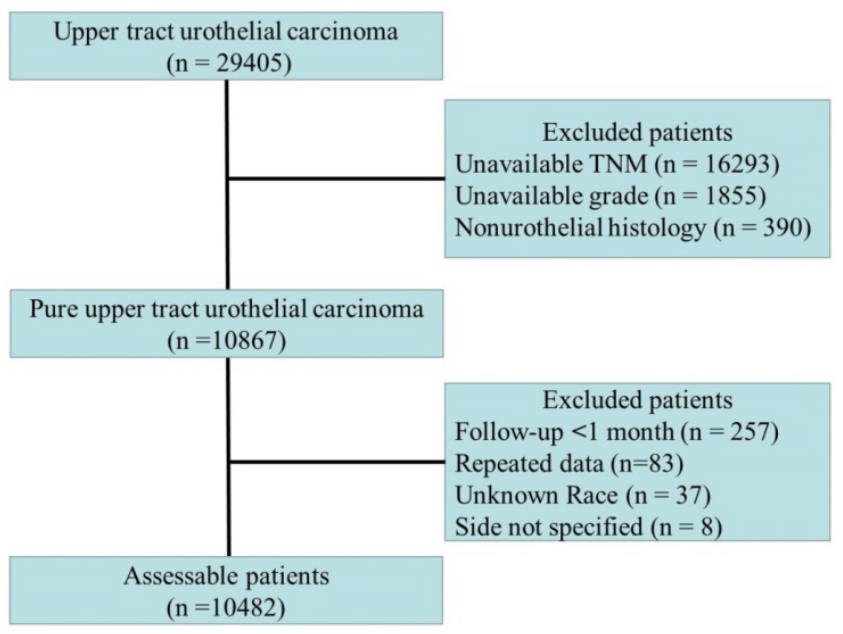

Figure 1. Flow chart of patients included in the primary analysis.

\section{Results}

A total of 10,482 eligible patients were identified; 2428 patients $(23.2 \%)$ died of UTUC, and 3295 patients (31.4\%) died of other causes (2004 to 2015) (Table 1). There were 6251 men (59.6\%) and 4231 women $(40.4 \%)$ included in the study, with a median age of 74 yrs. Overall, 6418 (61.2\%), 4051 (38.6\%) and 13 (0.1\%) patients had renal pelvic, ureteral and multifocal tumors, respectively. Lymph node removal was performed in 2711 patients $(25.9 \%)$. The proportion of patients with nodal metastases was $13.5 \%$ $(1414 / 10,482)$. The detailed clinicopathological characteristics of the cohort are listed in Table 1.

The median follow-up duration was 27 (1-143) months. The 5-year CSS of UTUC patients was $69.6 \%$ (68.4-70.8\%). Fig. 2 shows the CSS in UTUC patients. Mortality was significantly correlated with sex, race, tumor location, $\mathrm{T}$ stage, $\mathrm{N}$ stage, $\mathrm{M}$ stage, histologic grade and prognostic group (all $P<0.001$, Table 2). Interestingly, patients with N1 disease and N2 disease had similar mortality rates (5-year CSS: $32.8 \%$ vs. $33.2 \%, P=0.525$, Fig. 3A). With regard to grade (G) classification, patients with G1 and G2 disease had similar mortality rates $(P=0.127)$ to those of patients with $\mathrm{G} 3$ and $\mathrm{G} 4$ disease $(P=0.846)$ (Fig. 3B).

According to the AJCC-TNM prognostic stage group criteria, the 5-year CSS rates were $87.3 \%(95 \%$ CI $85.9 \%$ to $88.7 \%$ ), $79.3 \%$ (95\% CI $76.9 \%$ to $81.7 \%$ ), $64.9 \%(95 \%$ CI $62.7 \%$ to $67.1 \%$ ) and $32.8 \%$ (95\% CI $30.1 \%$ to $35.5 \%$ ) in patients with stage I to IV disease, respectively (I vs. II, $P<0.001$; II vs. III, $P<0.001$; III vs. IV $, P<0.001, P<0.001$, Fig. 4A).

However, among the patients with stage IV disease, 493 (22.5\%) had T4N0M0, 597 (27.2\%) had TxN1M0, 424 (19.4\%) had TxN2M0, and 677 (30.9\%) 
had metastases (TxNxM1). Additionally, the mortality curves for the T4N0M0, TxN1M0 and TxN2M0 stage groups overlapped and crossed. The 5-year CSS rates for patients with stage IV disease classified as T4N0M0, TxN1M0, TxN2M0 and TxNxM1 were $41.1 \%$ (95\% CI $35.2 \%$ to $47.0 \%$ ), $38.6 \%$ (95\% CI $33.1 \%$ to $44.1 \%), 40.4 \%$ (95\% CI $33.0 \%$ to $47.8 \%)$ and $14.2 \%$ (95\% CI $9.9 \%$ to $18.5 \%$ ), respectively (T4N0M0 vs. TxNxM1， $P<0.001 ;$ TxN1M0 vs. TxNxM1， $P<0.001$; TxN2M0 vs. TxNxM1, $P<0.001$, Fig. 3C). No significant differences in CSS were found among the patients with $\geq 2$ site-specific metastases $(n=73)$, single bone metastases $(n=60)$, single lung metastases $(n=89)$ and single liver metastases $(n=45)(P>0.10)$.

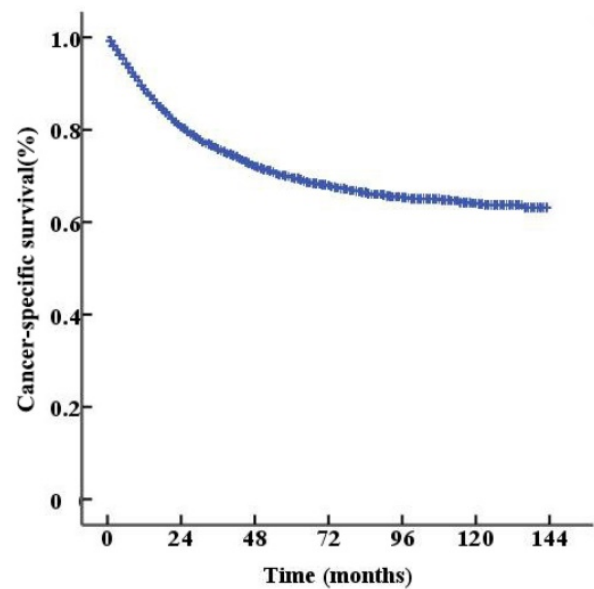

$\neg$ All patients(n=10482)

Figure 2. Kaplan-Meier cancer-specific survival (CSS) curves of patients with invasive upper tract urothelial carcinoma.

Stage IV tumors were subdivided on the basis of mortality data (Modified Stage 1): stage IVa tumors were considered nonmetastatic (T4NxM0 and TxN1-2M0; $\mathrm{n}=1514$ ), and stage $\mathrm{IVb}$ tumors were considered metastatic (TxNxM1, n=677). The 5-year CSS rates for patients with stage IVa and IVb disease were $39.9 \%$ (95\% CI $36.4 \%$ to $43.4 \%$ ) and $14.2 \%$ (95\% CI $9.9 \%$ to $18.5 \%)$, respectively $(P<0.001)$. These definitions provided improved prognostic stratification with significant differences in mortality between the modified categories (Fig. 4B).

Stage IV tumors were further subdivided (Modified Stage 2): stage IVa tumors were considered low grade (T4NxM0, TxN1-2M0, TxNxM1; G1-2; $\mathrm{n}=141)$, and stage $\mathrm{IVb}$ tumors were considered metastatic (T4NxM0, TxN1-2M0, TxNxM1; G3-4; $\mathrm{n}=2050$ ). The 5-year CSS rates for patients with stage IVa and IVb disease were $76.3 \%$ (95\% CI $68.7 \%$ to $83.9 \%)$ and $31.4 \%$ (95\% CI $28.5 \%$ to $34.3 \%)$, respectively $(P<0.001$, Fig. 3D). These definitions provided improved prognostic stratification with significant differences in mortality between the modified categories (Fig. 4C).
Table 1. Clinical and pathological characteristics of patients with upper tract urothelial carcinoma

\begin{tabular}{ll}
\hline Variable & Entire cohort $(\mathrm{n}=10482)$ \\
\hline Age (y) & 72.3 \\
Mean & 74.0 \\
Median & $22.0-101.0$ \\
Range & \\
Sex & $6251(59.6)$ \\
Male & $4231(40.4)$ \\
Female & \\
Race & $9230(88.1)$ \\
Caucasian & $1252(11.9)$ \\
Other & \\
T stage & $3557(33.9)$ \\
T1 & $1941(18.5)$ \\
T2 & $3962(37.8)$ \\
T3 & $1022(9.8)$ \\
T4 & \\
N stage & $9066(86.5)$ \\
N0 & $818(7.8)$ \\
N1 & $598(5.7)$ \\
N2 & \\
M stage & $9805(93.5)$ \\
M0 & $677(6.5)$ \\
M1 & \\
Grade & $2109(20.1)$ \\
G1-2 & $8373(79.8)$ \\
G3-4 &
\end{tabular}

Table 2. The 5-year cancer-specific mortality in patients with upper tract urothelial carcinoma

\begin{tabular}{lll}
\hline Variable & 5-year CSS & $P$ value \\
\hline Age (y) & & \\
Mean & & \\
Median & & \\
Range & & $<0.001$ \\
Sex & $77.9(70.7-73.5)$ & \\
Male & $66.0(64.2-67.8)$ & \\
Female & & $<0.001$ \\
Race & $70.5(69.3-71.7)$ & \\
Caucasian & $63.1(59.8-66.4)$ & \\
Other & & $<0.001$ \\
T stage & $84.7(83.3-86.1)$ & \\
T1 & $77.2(74.8-79.6)$ & \\
T2 & $59.2(67.2-61.2)$ & \\
T3 & $29.4(25.3-33.5)$ & \\
T4 & & $<0.001$ \\
N stage & $74.4(73.2-75.6)$ & \\
N0 & $32.8(28.3-37.3)$ & \\
N1 & $33.2(27.1-39.3)$ & \\
N2 & & $<0.001$ \\
M stage & $72.6(71.4-73.8)$ & \\
M0 & $14.2(9.9-18.5)$ & \\
M1 &
\end{tabular}




\begin{tabular}{lll}
\hline Variable & 5-year CSS & $P$ value \\
\hline Grade & $87.0(85.2-88.8)$ & $<0.001$ \\
G1-2 & $64.4(63.0-65.8$ & \\
G3-4 & & $<0.001$ \\
Tumor location & $72.4(70.6-74.2)$ & \\
Renal pelvis & $67.8(67.6-78.0)$ & \\
Ureter & & \\
Both & & \\
Laterality & $68.9(67.3-70.5)$ & \\
Right & $70.3(68.7-71.9)$ & \\
Left & & \\
Bilateral & & \\
Prognostic groups & $87.3(85.9-88.7)$ & \\
I & $79.3(76.9-81.7)$ & \\
II & $64.9(62.7-67.1)$ & \\
III & $32.8(30.1-35.5)$ & \\
IV &
\end{tabular}

Table 3. Predictive accuracy of the staging system

\begin{tabular}{lll}
\hline Stage & C-index & Bootstrap C-index \\
\hline AJCC-TNM & 0.73 & 0.72 \\
Modification 1 & 0.74 & 0.74 \\
Modification 2 & 0.73 & 0.73 \\
\hline
\end{tabular}

Harrell's concordance index (C-index) and bootstrap-corrected C-index values for the stages are shown in Table 3. The value of the modifications did
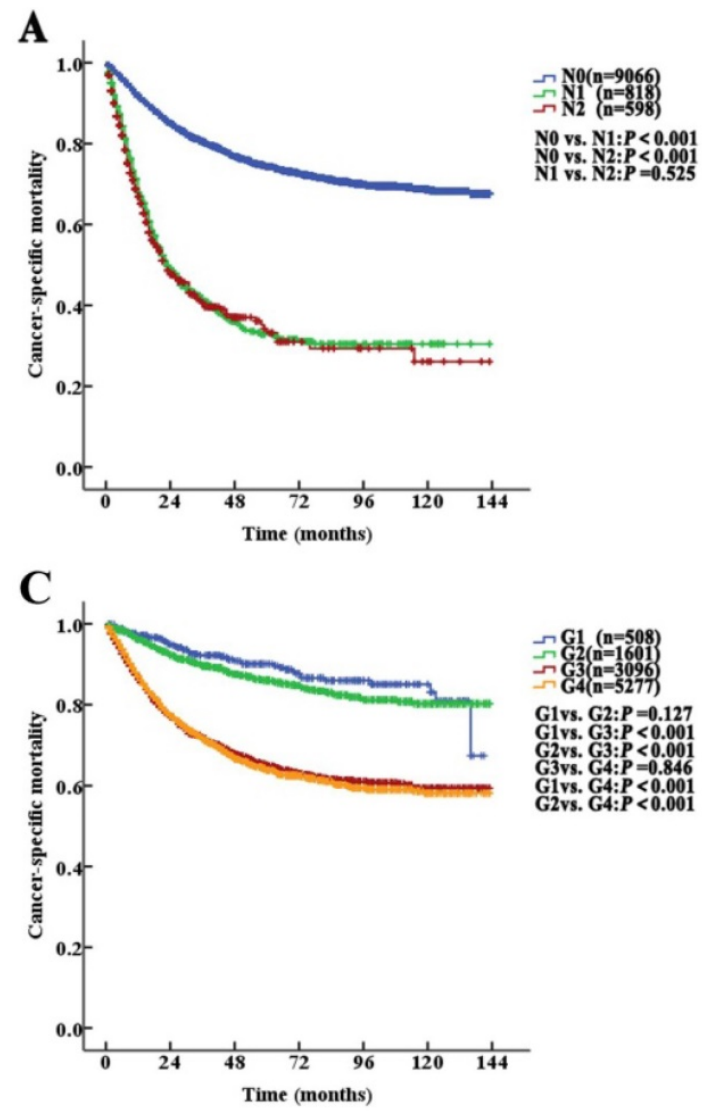

not decrease.

\section{Discussion}

To enable prognostic prediction, the AJCC/ UICC-TNM system was established using a mix of clinical and pathologic diagnoses, and it accurately reflects the prognosis in patients with UTUC [7]. The TNM classification is widely used to determine prognosis and inform the selection of an effective treatment. In the current AJCC-TNM prognostic group staging system, stage IV is defined as tumor invasion of the adjacent organs or through the kidney into the perinephric fat, lymph node metastasis and distant metastasis [2, 7]. However, the stage IV criteria are similar to those of previous versions without any recent changes $[10,11]$. In this study, we critically analyzed the heterogeneity of the stage IV criteria and discussed the predictive value and feasibility of a staging system for invasive UTUC. Based on different pathological factors, two modifications were proposed to help better differentiate AJCC stage IV disease without any loss of predictive value.

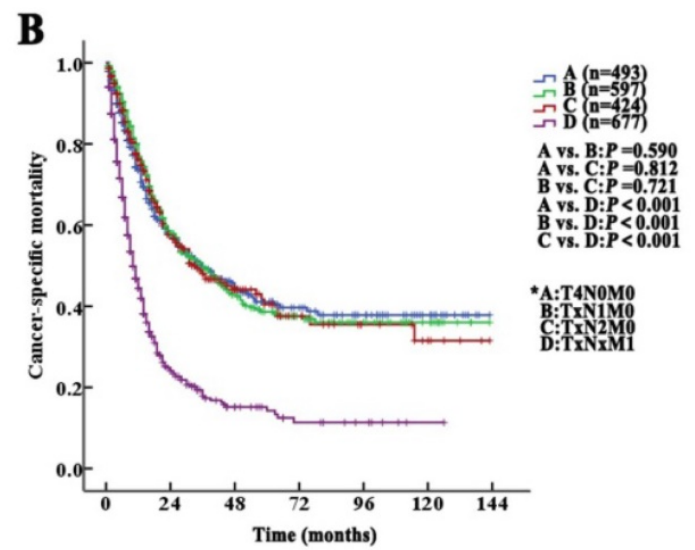

D

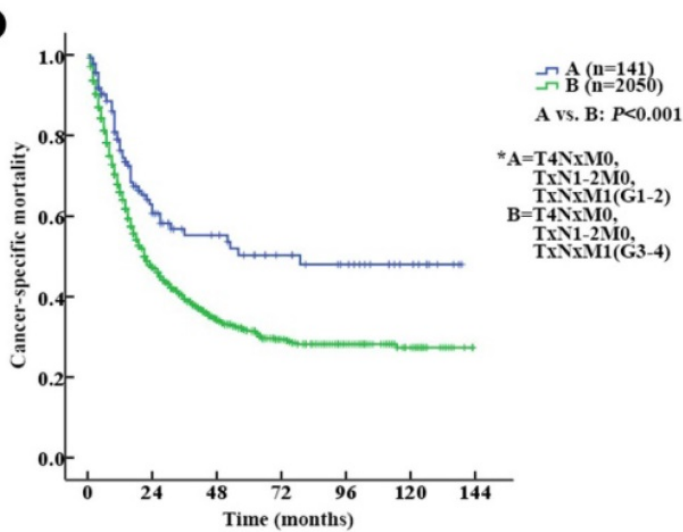

Figure 3. Kaplan-Meier cancer-specific survival (CSS) curves of patients with invasive upper tract urothelial carcinoma at different $N$ stages and grades. A. Stratification by $N$ stages, the 5-year CSS curves show no significant difference between patients with N1 and N2 disease. B. Stratification by stage IV of the AJCC-TNM prognostic group staging system with nonmetastatic or metastatic disease ( $A=T 4 N 0 M 0 ; B=T x N 1 M 0 ; C=T x N 2 M 0$ and $D=T x N x M 1)$. C. Stratification by grade, the 5 -year $C S S$ curves also show no significant difference between patients with $G 1$ and $G 2$ disease or between patients with $G 3$ and G4 disease. D. Stratification by stage IV of the AJCC-TNM prognostic group staging system with low or high grade (A=T4NxM0, TxN1-2M0, TxNxM1; G1-2; B=T4NxM0, TxN1-2M0, TxNxM1; G3-4). 
A

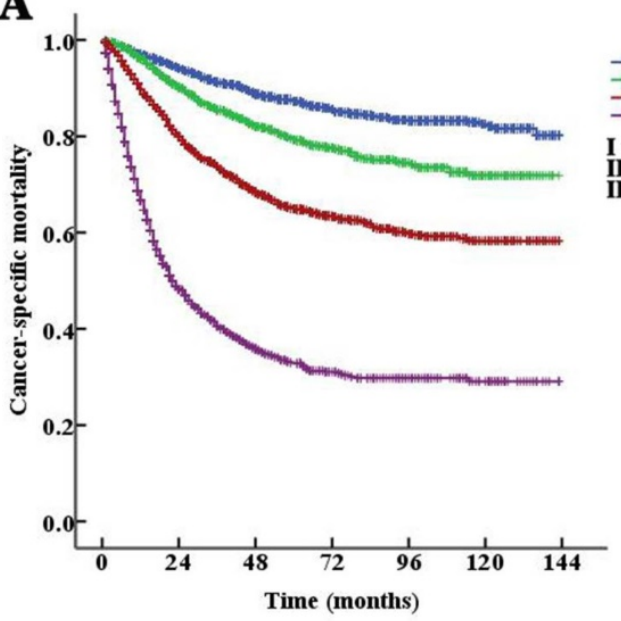

B

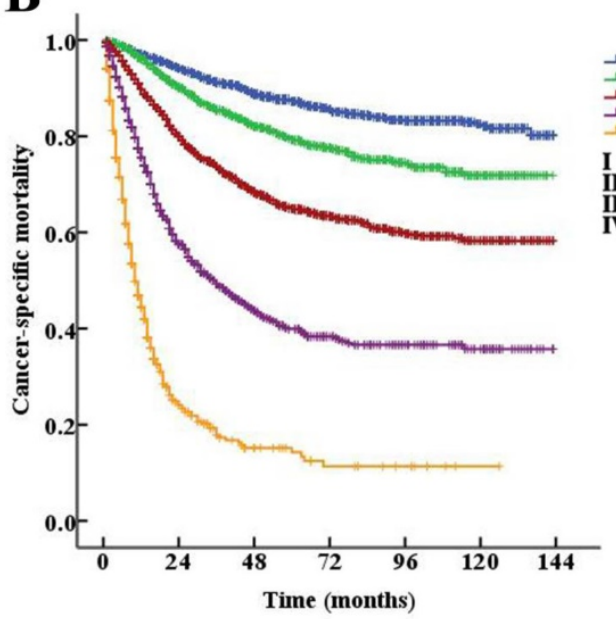

C

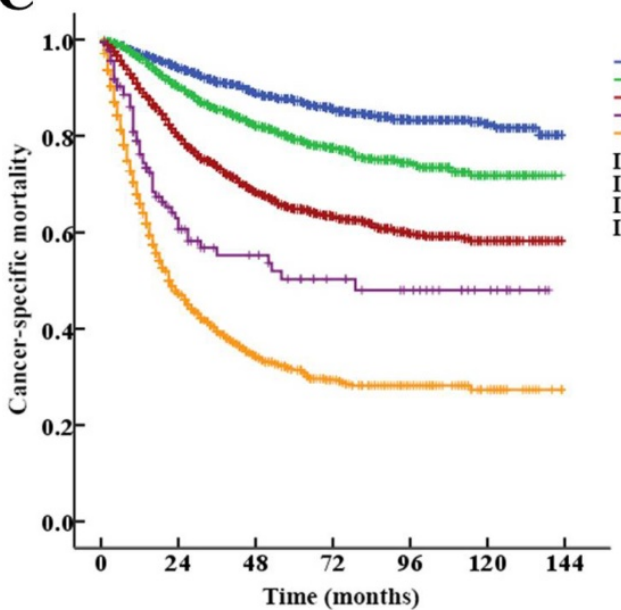

Figure 4. Kaplan-Meier cancer-specific survival (CSS) curves of patients with invasive upper tract urothelial carcinoma according to the AJCC-TNM prognostic group staging system. A. Stratification by the AJCC-TNM prognostic group staging system. B. Stratification by Modified Stage 1. IVa tumors were classified as nonmetastatic ( $\mathrm{T} 4 \mathrm{~N} \times \mathrm{MO}$ and $\mathrm{TxN1}-2 \mathrm{MO}$ ), and stage IVb tumors were classified as metastatic (TxNxM1). C. Stratification by Modified Stage 2. IVa tumors were classified as low-grade (T4NxM0, TxNI-2M0, TxNxMI; GI-2), and stage IVb tumors were classified as high-grade (T4NxM0, TxN1-2M0, TxNxM1; G3-4).

The prognosis of UTUC varies with stage. The extent of a tumor or metastasis is a confirmed prognostic factor $[12,13]$. Tumor stage is one of the most important predictors of survival in patients with UTUC [11, 14, 15]. Additionally, the involvement of the lymph nodes in UTUC is an independent predictive factor for poor survival [16, 17]. The EAU guidelines also affirm that postoperative factors, including tumor stage, lymph node involvement, and ureteral and/or multifocal tumors, affect prognosis [3]. Our study showed that the 5-year CSS rates for patients with stage I, II, III and IV disease were $87.3 \%$ (95\% CI $85.9 \%$ to $88.7 \%$ ), $79.3 \%$ (95\% CI $76.9 \%$ to $81.7 \%$ ), $64.9 \%$ (95\% CI $62.7 \%$ to $67.1 \%$ ) and $32.8 \%$ (95\% CI $30.1 \%$ to $35.5 \%$ ), respectively (I vs. II, $P<0.001$; II vs. III, $P<0.001$; III vs. IV, $P<0.001$, all: $P<0.001$ ).

Tumor grade is also one of the most important predictors of survival in patients with UTUC [18]. The updated World Health Organization Classification provides more useful insights into and approaches to individual categories $[6,19,20]$. In our study, stage IV tumors were subdivided as follows (Modified Stage 2): stage IVa tumors were considered low grade (T4NxM0, TxN1-2M0, TxNxM1; G1-2), and stage IVb tumors were considered metastatic (T4NxM0, TxN1-2M0, TxNxM1; G3-4), and there was a significant difference in mortality.

UTUC shares a similar etiology and histologic distribution with urothelial cancers of the bladder [19]. According to the AJCC prognostic stage for tumors of the urinary bladder, stage IV was subdivided into stage IVa (T4bN0M0 and TxNxM1a) and stage $\mathrm{IVb}(\mathrm{TxNxM} 1 \mathrm{~b})$ on the basis of the presence of distant metastases [21]. Stage IV UTUC tumors also include T4N0M0, TxN1M0, TxN2M0 and TxNxM1. Interestingly, we found that the stage IV subgroups were heterogeneous. The mortality curves for T4N0M0, TxN1M0 and TxN2M0 overlapped and crossed. In addition, M1 disease was generally associated with a poor prognosis. Further analysis, divided stage IV upper urinary tract carcinomas into locoregionally advanced and metastatic disease subcategories. The molecular mechanisms underlying the observed interaction between locally advanced disease and distant metastases in UTUC patients may help clarify the results of this study [8].

There are few accurate predictive tools for UTUC [3]. Nomograms, which use demographic and clinicopathologic data to predict oncologic outcomes, have attracted increased interest in the past few years [22-24]. Nomogram models have been constructed using different factors, so there is no consensus yet, and they are not widely used in clinical practice. Currently, AJCC prognostic stage groups are used to discriminate between prognostic subgroups within a patient population [25]. The treatment of UTUC differs significantly based on stage [26-28]. Our study 
suggests a subgroup analysis is useful in the stage IV group to establish more individualized therapeutic strategies and prognostic predictions. It is conceivable that the inclusion of detailed pathologic information could further enhance the individuation of the current model.

We acknowledge that our study had some limitations. First, the analyses were retrospective in nature because the collection of data from the SEER database was retrospective. The treating physician's perception of the patient's prognosis and the treatment (especially adjuvant therapies) were not included in the data. Second, other variables may be beneficial for predicting CSS. Unfortunately, the upper urinary tract tumor location, tumor architecture, tumor size, presence of lymphovascular invasion, molecular biology (chromosome abnormalities), biomarkers, and lymph node dissection could not be obtained from the SEER database. We did not calculate the difference with regard to established modifications because some factors could not be collected. We compared the newly developed modifications with the 8th AJCC-TNM classification system. The predictive accuracy of the modified AJCC-TNM prognostic group staging system should be tested in an external cohort to determine its validity with regard to prediction in clinical practice. Third, we failed to contribute any original laboratory finding or clinical observation from patients for external verification. However, we believe that different established modifications could be verified with unified data. Thus, all analyses should be considered exploratory rather than hypothesis-driven. Despite these limitations, we believe that a simple analysis is important to establish a basis for future validation studies with larger multicenter datasets.

Notwithstanding the above limitations, the current AJCC-TNM prognostic group staging system has room for improvement. Stage IV was stratified into two prognostically different risk groups depending on metastasis or grade. Revisions to the current AJCC-TNM prognostic group staging system may make it more concise and provide more detailed information to patients with invasive UTUC.

\section{Abbreviations}

AJCC/UICC: American Joint Committee on Cancer/Union Internationale Contre le Cancer; AUC: area under the curve; CSS: cancer-specific survival; EAU: European Association of Urology; NCCN: National Comprehensive Cancer Network; SEER: Surveillance, Epidemiology and End Results; TNM: tumor-node-metastasis; UTUC: upper tract urothelial carcinoma.

\section{Acknowledgements}

We are grateful to the authors, the enrolled patients, their families, and the referring physicians. We also gratefully acknowledge the contribution of all colleagues to this study. Additionally, we thank our families for their support.

\section{Funding}

This work was supported by the National Natural Science Foundation of China (Grant No. 81902610) and Science and Technology Planning Project of Shenzhen Municipality (CN) (JCYJ20190807145409328).

\section{Availability of data and materials}

The datasets used and/or analyzed during the present study are available from the corresponding author on reasonable request.

\section{Author contributions}

KF Xiao had full access to all data in the study and takes responsibility for the integrity of the data and the accuracy of the data analysis. Study conception and design: ZS Li, XY Li, and KF Xiao. Acquisition of data: All authors. Analysis and interpretation of data: All authors. Drafting of the manuscript: ZS Li and XY Li. Critical revision of the manuscript for important intellectual content: KF Xiao. Statistical analysis: All authors. Obtaining funding: ZS Li. Administrative, technical, or material support: KF Xiao. Supervision: ZS Li, XY Li, and KF Xiao. Other (specify): None.

\section{Ethics approval and consent to participate}

The present study was approved by the Ethics Committee of Shenzhen People's Hospital (No. LL-KY-2020123).

\section{Competing Interests}

The authors have declared that no competing interest exists.

\section{References}

1. Siegel RL, Miller KD, Jemal A. Cancer statistics, 2019. CA Cancer J Clin. 2019; 69: 7-34.

2. Koh WJ, Abu-Rustum NR, Bean S, Bradley K, Campos SM, Cho KR, et al. Uterine Neoplasms, Version 1.2018, NCCN Clinical Practice Guidelines in Oncology. J Natl Compr Canc Netw. 2018; 16: 170-99.

3. Roupret M, Babjuk M, Comperat E, Zigeuner R, Sylvester RJ, Burger M, et al. European Association of Urology Guidelines on Upper Urinary Tract Urothelial Carcinoma: 2017 Update. Eur Urol. 2018; 73: 111-22.

4. Ehdaie B, Shariat SF, Savage C, Coleman J, Dalbagni G. Postoperative nomogram for disease recurrence and cancer-specific death for upper tract urothelial carcinoma: comparison to American Joint Committee on Cancer staging classification. Urol J. 2014; 11: 1435-41.

5. Lee D, Yoo S, You D, Hong B, Cho YM, Hong JH, et al. Lymph node density vs. the American Joint Committee on Cancer TNM nodal staging system in node-positive bladder cancer in patients undergoing extended or super-extended pelvic lymphadenectomy. Urol Oncol. 2017; 35: 151 e1- e7.

6. Roupret M, Babjuk M, Burger M, Capoun O, Cohen D, Comperat EM, et al. European Association of Urology Guidelines on Upper Urinary Tract Urothelial Carcinoma: 2020 Update. Eur Urol. 2020; 20: 30427-9. 
7. Brierley. JD, Gospodarowicz MK, C. W. Comments on the TNM classification of malignant tumours--8th edition: Oxford, UK: John Wiley \& Sons Publisher. 2017.

8. Abdel-Rahman O. Revisiting the Prognostic Heterogeneity of AJCC Stage IV Carcinomas of the Upper Urinary Tract. Clin Genitourin Cancer. 2018; 16: e859-e65.

9. Abdel-Rahman O. Validation of the Eighth AJCC New Substages for Bladder Cancer Among Different Staging Contexts. Clin Genitourin Cancer. 2017; 15: e1095-e106.

10. Novak J, Fabian P. Comments on the TNM classification of malignant tumours - $7^{\text {th }}$ edition. Klin Onkol. 2011; 24: 149-50.

11. Lughezzani G, Burger M, Margulis V, Matin SF, Novara G, Roupret M, et al. Prognostic factors in upper urinary tract urothelial carcinomas: a comprehensive review of the current literature. Eur Urol. 2012; 62: 100-14.

12. Fa Yyazi A, Karimzadeh P, Torabian S, Damadi S, Khajeh A. Comparison of intravenous midazolam drip with intermittent intravenous diazepam in the treatment of refractory serial seizures in children. Iran J Child Neurol. 2012; 6: 15-9.

13. Ouzzane A, Colin P, Xylinas E, Pignot G, Ariane MM, Saint F, et al. Ureteral and multifocal tumours have worse prognosis than renal pelvic tumours in urothelial carcinoma of the upper urinary tract treated by nephroureterectomy. Eur Urol. 2011; 60: 1258-65.

14. Png KS, Lim EK, Chong KT, Sugiono M, Yip SK, Cheng CW. Prognostic factors for upper tract transitional cell carcinoma: a retrospective review of 66 patients. Asian J Surg. 2008; 31: 20-4.

15. Roupret M, Babjuk M, Comperat E, Zigeuner R, Sylvester RJ, Burger M, et al. European Association of Urology Guidelines on Upper Urinary Tract Urothelial Cell Carcinoma: 2015 Update. Eur Urol. 2015; 68: 868-79.

16. Roscigno M, Brausi M, Heidenreich A, Lotan Y, Margulis V, Shariat SF, et al. Lymphadenectomy at the time of nephroureterectomy for upper tract urothelial cancer. Eur Urol. 2011; 60: 776-83.

17. Petrelli F, Yasser Hussein MI, Vavassori I, Barni S. Prognostic Factors of Overall Survival in Upper Urinary Tract Carcinoma: A Systematic Review and Meta-analysis. Urology. 2017; 100: 9-15.

18. Flaig TW. NCCN Guidelines Updates: Management of Muscle-Invasive Bladder Cancer. J Natl Compr Canc Netw. 2019; 17: 591-3.

19. Wang G, McKenney JK. Urinary Bladder Pathology: World Health Organization Classification and American Joint Committee on Cancer Staging Update. Arch Pathol Lab Med. 2019; 143: 571-7.

20. Gallan AJ, Choy B, Paner GP. Contemporary Grading and Staging of Urothelial Neoplasms of the Urinary Bladder: New Concepts and Approaches to Challenging Scenarios. Surg Pathol Clin. 2018; 11: 775-95.

21. Nishiyama H. Asia Consensus Statement on NCCN Clinical Practice Guideline for bladder cancer. Jpn J Clin Oncol. 2018; 48: 3-6.

22. Cha EK, Shariat SF, Kormaksson M, Novara G, Chromecki TF, Scherr DS, et al. Predicting clinical outcomes after radical nephroureterectomy for upper tract urothelial carcinoma. Eur Urol. 2012; 61: 818-25.

23. $\mathrm{Ku} \mathrm{JH}$, Moon $\mathrm{KC}$, Jung JH, Jeong $\mathrm{SH}$, Kwak C, Kim HH. External validation of an online nomogram in patients undergoing radical nephroureterectomy for upper urinary tract urothelial carcinoma. British journal of cancer. 2013; 109 : 1130-6.

24. Krabbe LM, Eminaga O, Shariat SF, Hutchinson RC, Lotan Y, Sagalowsky AI, et al. Postoperative Nomogram for Relapse-Free Survival in Patients with High Grade Upper Tract Urothelial Carcinoma. J Urol. 2017; 197: 580-9.

25. Zivanovic $\mathrm{O}$, Leitao $\mathrm{MM}$, Iasonos $\mathrm{A}$, Jacks $\mathrm{LM}$, Zhou $\mathrm{O}$ Abu-Rustum NR, et al. Stage-specific outcomes of patients with uterine leiomyosarcoma: a comparison of the international Federation of gynecology and obstetrics and american joint committee on cancer staging systems. Journal of clinical oncology : official journal of the American Society of Clinical Oncology. 2009; 27: 2066-72.

26. Kubota Y, Hatakeyama S, Tanaka T, Fujita N, Iwamura H, Mikami J, et al. Oncological outcomes of neoadjuvant chemotherapy in patients with locally advanced upper tract urothelial carcinoma: a multicenter study. Oncotarget. 2017; 8: 101500-8.

27. Seisen T, Krasnow RE, Bellmunt J, Roupret M, Leow JJ, Lipsitz SR, et al. Effectiveness of Adjuvant Chemotherapy After Radical Nephroureterectomy for Locally Advanced and/or Positive Regional Lymph Node Upper Tract Urothelial Carcinoma. Journal of clinical oncology : official journal of the American Society of Clinical Oncology. 2017; 35: 852-60.

28. Moschini M, Shariat SF, Roupret M, De Santis M, Bellmunt J, Sternberg CN, et al. Impact of Primary Tumor Location on Survival from the European Organization for the Research and Treatment of Cancer Advanced Urothelial Cancer Studies. J Urol. 2018; 199: 1149-57. 